This is the author's final, peer-reviewed manuscript as accepted for publication. The publisher-formatted version may be available through the publisher's web site or your institution's library.

\title{
Creating materials with a desired refraction coefficient: numerical experiments
}

Sapto W. Indratno and Alexander G. Ramm

How to cite this manuscript (APA format)

If you make reference to this version of the manuscript, use the following citation format:

Indratno, S.W., Ramm, A.G. (2010). Creating materials with a desired refraction coefficient: numerical experiments. Retrieved from http://krex.ksu.edu

\section{Published Version Information}

Citation: Indratno, S.W., Ramm, A.G. (2010). Creating materials with a desired refraction coefficient: numerical experiments. International Journal of Computing Science and Mathematics, 3 (1/2), 76-101

Copyright: Copyright $@$ 2004-2011 Inderscience Enterprises Limited.

Digital Object Identifier (DOI): 10.1504/IJCSM.2010.033928

Publisher's Link:

http://www.inderscience.com/search/index.php?action=record\&rec_id=33928\&prevQuer $y=\& p s=10 \& m=0 r$

This item was retrieved from the K-State Research Exchange (K-REx), the institutional repository of Kansas State University. K-REx is available at http://krex.ksu.edu 


\title{
Creating materials with a desired refraction coefficient: numerical experiments
}

\section{Sapto W. Indratno and Alexander G. Ramm*}

\author{
Department of Mathematics, \\ Kansas State University, \\ Manhattan, KS 66506-2602, USA \\ E-mail: sapto@math.ksu.edu \\ E-mail: ramm@math.ksu.edu \\ ${ }^{*}$ Corresponding author
}

\begin{abstract}
A recipe for creating materials with a desired refraction coefficient is implemented numerically. An error estimate is given for the approximate solution of the many-body scattering problem in the case of small scatterers. This result is used for the estimate of the minimal number of small particles to be embedded in a given domain $D$ in order to get a material whose refraction coefficient approximates the desired one with the relative error not exceeding a desired small quantity.
\end{abstract}

Keywords: many-body wave scattering problem; metamaterials; refraction coefficient.

Reference to this paper should be made as follows: Indratno, S.W. and Ramm, A.G. (2010) 'Creating materials with a desired refraction coefficient: numerical experiments', Int. J. Computing Science and Mathematics, Vol. 3, Nos. 1/2, pp.76-101.

Biographical notes: Sapto W. Indratno is currently a $\mathrm{PhD}$ student at Kansas State University under the supervision of Prof. Alexander G. Ramm. He is a co-author of four accepted papers. His fields of interest are numerical analysis, optimisation, stochastic processes, inverse and ill-posed problems, scattering theory, differential equations and applied mathematics.

\begin{abstract}
Alexander G. Ramm is an author of more than 590 papers, 2 patents, 12 monographs, an Editor of 3 books. He is an Associate Editor of several journals. He gave more than 140 addresses at various conferences, visited many Universities in Europe, Asia, Australia and USA. He won Khwarizmi International Award in Mathematics, was a London Mathematical Society Speaker, distinguished HKSTAM Speaker, CNRS Research Professor, Fulbright Professor in Israel, distinguished Foreign Professor in Mexico and Egypt. His research interests include many areas of analysis, numerical analysis and mathematical physics.
\end{abstract}




\section{Introduction}

A theory of wave scattering by many small bodies embedded in a bounded domain $D$ filled with a material with known refraction coefficient was developed in Ramm (2007a, 2007b, 2008a, 2008b). It was assumed in Ramm (2007a) that

$$
d=O\left(a^{1 / 3}\right), \quad M=O\left(a^{-1}\right), \quad \frac{\partial u_{M}}{\partial \nu}=\zeta_{m} u_{M} \quad \text { on } S_{m}, \quad 1 \leq m \leq M,
$$

where $a$ is the characteristic size of the small particles, $d$ is the distance between two neighbouring particles, $M$ is the total number of the embedded particles, $S_{m}$ is the boundary of $m$ th particle $D_{m}, \nu$ is the unit normal to $S_{m}$ directed out of $D_{m}$, and $\zeta_{m}=h_{m} / a$, where $h_{m}, \operatorname{Im} h_{m} \leq 0,1 \leq m \leq M$, are constants independent of $a$.

Let us assume that $D$ is filled with a material with known refraction coefficient $n_{0}^{2}(x), \quad \operatorname{Im} n_{0}^{2}(x) \geq 0, \quad n_{0}^{2}(x)=1$ in $D^{\prime}:=\mathbb{R}^{2} \backslash D, n_{0}^{2}(x)$ is Riemann integrable. The governing equation is

$$
\begin{aligned}
& L_{0} u_{0}:=\left[\triangle+k^{2} n_{0}^{2}\right] u_{0}=0, \quad \text { in } \mathbb{R}^{3}, \\
& u_{0}=\exp (i k x \cdot \alpha)+v_{0}
\end{aligned}
$$

where $k$ is the wave number, $\alpha \in S^{2}$ is the direction of the incident plane wave, $S^{2}$ is the unit sphere in $\mathbb{R}^{3}$, and $v_{0}$ is the scattered field satisfying the radiation condition

$$
\lim _{r \rightarrow \infty} r\left(\frac{\partial v_{0}}{\partial r}-i k v_{0}\right)=0 \quad r:=|x| \rightarrow \infty,
$$

and the limit is attained uniformly with respect to the directions $x^{0}:=x / r$.

Let $n^{2}(x)$ be a desired refraction coefficient in $D$. We assume that $n^{2}(x)$ is Riemann integrable, $\operatorname{Im} n^{2}(x) \geq 0, n^{2}(x)=1$ in $D^{\prime}$. Our objective is to create materials with the refraction coefficient $n^{2}(x)$ in $D$ by embedding into $D$ many small non-intersecting balls $B_{m}, 1 \leq m \leq M$, of radius $a$, centred at some points $x_{m} \in D$. If one embeds $M$ small particles $B_{m}$ in the bounded domain $D$, then the scattering problem consists of finding the solution to the following problem:

$$
\begin{aligned}
& L_{0} u_{M}:=\left[\triangle+k^{2} n_{0}^{2}\right] u_{M}(x)=0 \quad x \in \mathbb{R}^{3} \backslash \cup_{m=1}^{M} B_{m}, \\
& \frac{\partial u_{M}}{\partial \nu}=\zeta_{m} u_{M} \quad \text { on } S_{m}:=\partial B_{m}, \quad 1 \leq m \leq M, \\
& u_{M}=u_{0}+v_{M},
\end{aligned}
$$

where $u_{0}$ solves problem (1)-(3), and $v_{M}$ satisfies the radiation condition.

The following theorem is proved in Ramm (2008b) under the assumptions

$$
\zeta_{m}=h\left(x_{m}\right) / a^{\kappa}, \quad d=O\left(a^{(2-\kappa) / 3}\right), \quad M=O\left(1 / a^{2-\kappa}\right), \quad \kappa \in(0,1),
$$

where $h(x)$ is a continuous function in $D, \operatorname{Im} h \leq 0, \kappa \in(0,1)$ is a parameter, and one can choose $h(x)$ and $\kappa$ as one wishes. Below it is always assumed that conditions (7) hold.

Theorem 1.1 (Ramm, 2008b): Assume that conditions (7) are satisfied, and $D_{m}$ is a ball of radius a centred at a point $x_{m}$. Let $h(x)$ in $(7)$ be an arbitrary continuous 
function in $D, \operatorname{Im} h(x) \leq 0, \Delta_{p} \subset D$ be any subdomain of $D$, and $\mathcal{N}\left(\Delta_{p}\right)$ be the number of particles in $\Delta_{p}$,

$$
\mathcal{N}\left(\Delta_{p}\right)=\frac{1}{a^{2-\kappa}} \int_{\Delta_{p}} N(x) d x[1+o(1)], \quad a \rightarrow 0,
$$

where $N(x) \geq 0$ is a given continuous function in $D$. Then

$$
\lim _{a \rightarrow 0}\left\|u_{e}(x)-u(x)\right\|_{C(D)}=0,
$$

where

$$
u_{e}(x):=u_{0}(x)-4 \pi \sum_{j=1}^{M} G\left(x, x_{j}\right) h\left(x_{j}\right) u_{e}\left(x_{j}\right) a^{2-\kappa}[1+o(1)], \quad a \rightarrow 0,
$$

where $\min _{j}\left|x-x_{j}\right| \geq a$, and $G(x, y)$ is the Green function of the operator $L_{0}$ in $\mathbb{R}^{3}, L_{0} G=-\delta(x-y)$ in $\mathbb{R}^{3}, G(x, y)$ satisfies the radiation condition. The numbers $u_{e}\left(x_{j}\right), 1 \leq j \leq M$, are found from the following linear algebraic system:

$$
\begin{gathered}
u_{e}\left(x_{m}\right)=u_{0}\left(x_{m}\right)-4 \pi \sum_{j=1, j \neq m}^{M} G\left(x_{m}, x_{j}\right) h\left(x_{j}\right) u_{e}\left(x_{j}\right) a^{2-\kappa}, \\
m=1,2, \ldots, M
\end{gathered}
$$

which is uniquely solvable for all sufficiently large M. The function

$$
u(x)=\lim _{a \rightarrow 0} u_{e}(x)
$$

solves the following limiting equation:

$$
u(x)=u_{0}(x)-\int_{D} G(x, y) p(y) u(y) d y,
$$

where $u_{0}$ satisfies equations (1)-(3),

$$
\begin{aligned}
& p(x):=4 \pi N(x) h(x), \\
& n^{2}(x):=1-k^{-2} q(x), \\
& q(x):=q_{0}(x)+p(x), \quad q_{0}(x):=k^{2}-k^{2} n_{0}^{2}(x),
\end{aligned}
$$

and $n_{0}^{2}(x)$ is the coefficient in (1).

In Ramm (2008b) a recipe for creating material with a desired refraction coefficient is formulated.

The goal of this paper is to implement numerically the recipe for creating materials with a desired refraction coefficient in a given domain $D$ by embedding in $D$ many small particles with prescribed physical properties. These particles are balls of radius $a$, centred at the points $x_{m} \in D$, and their physical properties are described 
by the boundary impedances $\zeta_{m}=h\left(x_{m}\right) / a^{\kappa}$. A formula for embedding the small balls in $D$ is given in Section 2.

We give an estimate for the error in the refraction coefficient of the medium obtained by embedding finitely many $(M<\infty)$ small particles, compared with the refraction coefficient of the limiting medium $(M \rightarrow \infty)$. This is important because in practice one cannot go to the limit $M \rightarrow \infty$, i.e., $a \rightarrow 0$, and one has to know the maximal $a$ (i.e., minimal $M$ ) such that the corresponding to this $a$ refraction coefficient differs from the desired refraction coefficient by not more than a given small quantity. In Section 3 we give an algorithm for finding the minimal number $M$ of the embedded small balls which generate a material whose refraction coefficient differs from a desired one by not more than a desired small quantity. In Section 4 some numerical experiments are described.

\section{Embedding small balls into a cube}

In this section we give a formula for distributing small balls in a cube in such a way that the second and third restrictions (7) are satisfied.

Without loss of generality let us assume that the domain $D$ is the unit cube:

$$
D:=[0,1] \times[0,1] \times[0,1] .
$$

Let

$$
D=\cup_{q=1}^{n^{3}} \overline{\Delta_{q}}, \quad n \in \mathbb{N}, \quad \Delta_{i} \cap \Delta_{j}=\emptyset \text { for } i \neq j,
$$

where $\mathbb{N}$ is the set of positive integers, $\bar{X}$ is the closure of the set $X$, and $\Delta_{q}$, $q=1,2, \ldots, n^{3}$, are cubes of side length $1 / n$.

Definition: We say that $D$ has property $Q_{n}$ if each small cube $\Delta_{q}$ contains a ball of radius $a_{n}, 0<a_{n}<1 / n$, centred at the centroid of the cube $\Delta_{q}$, and the following condition holds

$$
d_{n}:=\min _{q \neq j} \operatorname{dist}\left(B_{a_{n}}\left(x_{q}\right), B_{a_{n}}\left(x_{j}\right)\right)=\gamma a_{n}^{(2-\kappa) / 3},
$$

where $x_{q}$ is the centroid of the cube $\Delta_{q}, q=1,2, \ldots, n^{3}$,

$$
B_{a}(x):=\left\{y \in \mathbb{R}^{3}|| y-x \mid<a\right\},
$$

and $\gamma>0$ is a constant which is not too small (see formula (25)).

From (17) and (18) one gets

$$
d_{n}=l_{n}-2 a_{n}=\gamma a_{n}^{(2-\kappa) / 3}, \quad l_{n}:=1 / n .
$$

Since $l_{n}=1 / n$, the quantity $a_{n}$ solves the equation

$$
\gamma a^{(2-\kappa) / 3}+2 a-1 / n=0 .
$$


The function $f(a):=\gamma a^{(2-\kappa) / 3}+2 a$ is strictly growing on $[0, \infty)$. Thus, the solution to equation (21) exists, is unique, and can be calculated numerically, for example, by the bisection method.

However, it is easy to derive an analytic asymptotic formula for $a_{n}$ as $n \rightarrow \infty$. This formula is simple and can be used for all $n$ we are interested in, since these $n$ are sufficiently large.

Let us derive this asymptotic formula. Since $1 / 3<(2-\kappa) / 3<2 / 3$, one has $a \ll a^{(2-\kappa) / 3}$ if $a \ll 1$. Therefore,

$$
a_{n}=[1 /(n \gamma)]^{3 /(2-\kappa)}[1+o(1)], \quad \text { as } n \rightarrow \infty,
$$

is the desired asymptotic formula for the solution to (21). Note that

$$
\lim _{n \rightarrow \infty} a_{n}=0 \text { and } \lim _{n \rightarrow \infty} n a_{n}=0,
$$

as follows from (22) because $3 /(2-\kappa)>1$.

Note that $a_{n} / d_{n} \ll 1$, if $n \gg 1$, because (20) yields

$$
a_{n} / d_{n}=a_{n} /\left(\gamma a_{n}^{(2-\kappa) / 3}\right)=a_{n}^{(1+\kappa) / 3} / \gamma \ll 1 .
$$

Let us choose $n$ sufficiently large so that

$$
\gamma \gg\left(l_{n} / 2\right)^{(1+\kappa) / 3}, \quad \kappa \in(0,1), \quad l_{n}=1 / n,
$$

and make the following assumption:

Assumption A): The domain $D$ has property $Q_{m P}$.

This assumption means that the following conditions are satisfied:

$D=\cup_{q=1}^{P^{3}} \overline{\Omega_{q}}, \Omega_{j} \cap \Omega_{i}=\emptyset$ for $j \neq i$, where each cube $\Omega_{q}$ has side length $1 / P$, and $m^{3}$ small balls are embedded in $\Omega_{q}$ so that the following two conditions hold:

1 Each cube $\Omega_{q}$ is a union of small sub-cubes $\Delta_{j, q}$ :

$\Omega_{q}=\cup_{j=1}^{m^{3}} \Delta_{j, q}, \quad \Delta_{i, q} \cap \Delta_{j, q}=\emptyset$ for $i \neq j$,

where $\Delta_{j, q}, j=1,2, \ldots, m^{3}, q=1,2, \ldots, P^{3}$, are cubes of side length $1 /(m P)$,

2 In each sub-cube $\Delta_{j, q}$ there is a ball of radius $a_{m P}, 0<a_{m P}<1 /(m P)$, centred at the centroid of the sub-cube $\Delta_{j, q}$, and the radius $a_{m P}$ of the embedded balls satisfies the relation

$$
1 /(m P)-2 a_{m P}=\gamma a_{m P}^{(2-\kappa) / 3}, \quad \gamma \gg[1 /(2 m P)]^{(\kappa+1) / 3},
$$

where $\gamma>0$ is a fixed constant.

Lemma 2.2: If Assumption A) holds, then

$$
\lim _{m \rightarrow \infty} M a_{m P}^{2-\kappa}=1 / \gamma^{3},
$$


where $M=(m P)^{3}$ is the total number of small balls embedded in the unit cube $D$, $\gamma>0$ is fixed, and

$$
a_{m P}=[1 /(\gamma m P)]^{3 /(2-\kappa)}[1+o(1)] \text { as } m \rightarrow \infty .
$$

Proof: Relation (29) is an immediate consequence of (27). Using this relation, one obtains

$$
\begin{aligned}
\lim _{m \rightarrow \infty} M a_{m P}^{2-\kappa} & =\lim _{m \rightarrow \infty}(m P)^{3} a_{m P}^{2-\kappa} \\
& =\lim _{m \rightarrow \infty}(m P)^{3}[1 /(\gamma m P)]^{3}[1+o(1)] \\
& =\lim _{m \rightarrow \infty}\left(1 / \gamma^{3}\right)[1+o(1)]=1 / \gamma^{3} .
\end{aligned}
$$

Lemma 2.2 is proved.

\section{A recipe for creating materials with a desired refraction coefficient}

In this section the recipe given in Ramm (2008b) is used for creating materials with a desired refraction coefficient by embedding into $D$ small balls so that Assumption A) holds.

Step 1: Given the refraction coefficient $n_{0}^{2}(x)$ of the original material in $D$ and the desired refraction coefficient $n^{2}(x)$ in $D$, one calculates

$$
p(x)=k^{2}\left[n_{0}^{2}(x)-n^{2}(x)\right]=p_{1}(x)+i p_{2}(x),
$$

where

$$
p_{1}:=\operatorname{Re} p(x) \text { and } p_{2}(x):=\operatorname{Im} p(x)
$$

Choose

$$
N(x)=1 / \gamma^{3},
$$

where $\gamma$ is the constant $\gamma$ in Assumption A).

Step 2: Choose

$$
h(x)=h_{1}(x)+i h_{2}(x),
$$

where the functions $h_{1}(x)$ and $h_{2}(x)$ are defined by the formulas:

$$
h_{i}(x)=\gamma^{3} p_{i}(x) /(4 \pi), \quad i=1,2,
$$

and the functions $p_{i}(x)$ are defined in Step 1 .

Step 3: Partition $D$ into $P$ small cubes $\Omega_{p}$ with side length $1 / P$, and embed $m^{3}$ small balls in each cube $\Omega_{p}$ so that Assumption A) holds. 
Then

$$
\mathcal{N}\left(\Omega_{p}\right)=\frac{1}{a_{m P}^{2-\kappa}} \int_{\Omega_{p}} N(x) d x=\left|\Omega_{p}\right| /\left(\gamma^{3} a_{m P}^{2-\kappa}\right)=1 /\left[\gamma P a_{m P}^{(2-\kappa) / 3}\right]^{3}
$$

where $\mathcal{N}\left(\Delta_{p}\right)$ is the number of the balls embedded in the cube $\Omega_{p}, \kappa \in(0,1), a_{m P}$ is the radius of the embedded balls, and $\left|\Omega_{p}\right|$ is the volume of the cube $\Omega_{p}$.

Since

$$
a_{m P}=[1 /(m \gamma P)]^{\frac{3}{2-\kappa}}[1+o(1)] \text { as } m \rightarrow \infty
$$

it follows that

$$
\lim _{m \rightarrow \infty} \frac{\mathcal{N}\left(\Omega_{p}\right)}{m^{3}}=1
$$

By Assumption A) the balls are situated at the distances $\gamma a_{m P}^{\frac{2-\kappa}{3}}, \gamma>[1 /(m P)]^{\frac{1+\kappa}{3}}$. Therefore, all the assumptions, made in Theorem 1.1, hold. Thus,

$$
\max _{x \in D}\left|u_{e}(x)-u(x)\right| \rightarrow 0 \text { as } M \rightarrow \infty
$$

where $u_{e}(x)$ is defined in (10) and $u(x)$ solves (12). Let us assume for simplicity that $n_{0}^{2}(x)=1$, so that

$$
G(x, y)=g(x, y):=\exp (i k|x-y|) /(4 \pi|x-y|) .
$$

Then

$$
\begin{aligned}
u_{e}(x) & =u_{0}(x)-4 \pi \sum_{j=1}^{M} g\left(x, x_{j}\right) h\left(x_{j}\right) u_{e}\left(x_{j}\right) a_{m P}^{2-\kappa}, \quad\left|x-x_{j}\right|>a_{m P}, \\
M & :=(m P)^{3}
\end{aligned}
$$

and the limiting function

$$
u(x)=\lim _{M \rightarrow \infty} u_{e}(x)
$$

solves the integral equation

$$
u(x)+T u(x)=u_{0}(x)
$$

where

$$
\begin{aligned}
T u(x) & :=\int_{D} g(x, y) p(y) u(y) d y, \\
g(x, y) & :=\exp (i k|x-y|) /(4 \pi|x-y|),
\end{aligned}
$$


$M:=(m P)^{3}, h(x)=h_{1}(x)+i h_{2}(x), h_{i}(x), i=1,2$, are defined in (34),

$$
\begin{aligned}
p(x) & =k^{2}\left[n_{0}^{2}(x)-n^{2}(x)\right]=4 \pi\left[h_{1}(x)+i h_{2}(x)\right] N(x) \\
& =4 \pi\left[h_{1}(x)+i h_{2}(x)\right] / \gamma^{3},
\end{aligned}
$$

and the function $u_{0}(x)$ in (38) solves the scattering problem (1)-(3).

It follows from (37) that

$$
\max _{1 \leq l \leq M}\left|u\left(x_{l}\right)-u_{e}\left(x_{l}\right)\right| \rightarrow 0 \quad \text { as } M \rightarrow \infty
$$

where $u_{e}(x)$ and $u(x)$ are defined in (38) and (39), respectively. Here and throughout this paper $D:=\cup_{j=1}^{M} D_{j}, M:=(m P)^{3}, D_{j}(j=1,2, \ldots, M)$ are cubes with the side length $1 /(m P), D_{j} \cap D_{l}=\emptyset$ for $j \neq l$, and $x_{j}$ denotes the centre of the cube $D_{j}$. In the following paragraphs we derive the rate of convergence in (43). We denote

$$
\|u\|_{\infty}:=\sup _{x \in D}|u(x)| \text { and }\|v\|_{\mathbb{C}^{M}}:=\max _{1 \leq j \leq M}\left|v_{j}\right|, \quad v:=\left(\begin{array}{c}
v_{1} \\
v_{2} \\
\vdots \\
v_{M}
\end{array}\right) \in \mathbb{C}^{M},
$$

where $\mathbb{C}$ is the set of complex numbers.

Consider the following piecewise-constant function as an approximate solution to equation (39):

$$
u_{(M)}(x):=\sum_{j=1}^{M} \chi_{j}(x) u_{j, M},
$$

where $u_{j, M}(j=1,2, \ldots, M)$ are constants and

$$
\chi_{j}(x):= \begin{cases}1, & x \in D_{j} \\ 0, & \text { otherwise }\end{cases}
$$

Substituting $u_{(M)}(x)$ for $u(x)$ in (39) and evaluating at points $x_{l}$, one gets the following Linear Algebraic System (LAS) which is used to find the unknown $u_{j, M}$ :

$$
\tilde{u}_{(M)}+T_{d, M} \tilde{u}_{(M)}=u_{0, M},
$$

where $T_{d, M}$ is a discrete version of $T_{M}$, defined below,

$$
\tilde{u}_{(M)}:=\left(\begin{array}{c}
u_{1, M} \\
u_{2, M} \\
\vdots \\
u_{M, M}
\end{array}\right) \in \mathbb{C}^{M}, \quad u_{0, M}:=\left(\begin{array}{c}
u_{0}\left(x_{1}\right) \\
u_{0}\left(x_{2}\right) \\
\vdots \\
u_{0}\left(x_{M}\right)
\end{array}\right) \in \mathbb{C}^{M}
$$


$u_{0}(x)$ solves problem (1)-(3), and

$$
\left(T_{d, M} v\right)_{l}:=\sum_{j=1}^{M} \int_{D_{j}} g\left(x_{l}, y\right) p(y) d y v_{j}, \quad l=1,2, \ldots, M, \quad v:=\left(\begin{array}{c}
v_{1} \\
v_{2} \\
\vdots \\
v_{M}
\end{array}\right) \in \mathbb{C}^{M} .
$$

Multiplying the $l$ th equation in (46) by $\chi_{l}(x), l=1,2, \ldots, M$, and summing up over $l$ from 1 to $M$, one gets

$$
u_{(M)}(x)=u_{0,(M)}(x)-T_{M} u_{(M)}(x),
$$

where $u_{(M)}(x)$ is defined in (44),

$$
u_{0,(M)}(x):=\sum_{j=1}^{M} \chi_{j}(x) u_{0}\left(x_{j}\right),
$$

and

$$
T_{M} u(x):=\sum_{j=1}^{M} \chi_{j}(x) \int_{D} g\left(x_{j}, y\right) p(y) u(y) d y, \quad M=(m P)^{3} .
$$

It was proved in Ramm (2009) that equation (49) is equivalent to (46) in the sense that $\left\{u_{j, M}\right\}_{j=1}^{M}$ solves (46) if and only if function (44) solves (49).

Lemma 3.1: For all sufficiently large $M$ equation (46) has a unique solution, and there exists a constant $c_{1}>0$ such that

$$
\left\|\left(I_{d, M}+T_{d, M}\right)^{-1}\right\| \leq c_{1}, \quad \forall M>M_{0},
$$

where $M_{0}>0$ is a sufficiently large number.

Proof: Consider the operators $T$ and $T_{M}$ as operators in the space $L^{\infty}(D)$ with the sup-norm. Let $\left\|\left(T-T_{M}\right) u\right\|_{\infty}:=\sup _{x \in D}\left|\left(T-T_{M}\right) u(x)\right|$. Then

$$
\begin{aligned}
\left\|\left(T-T_{M}\right) u\right\|_{\infty} & \leq \max _{i} \sup _{x \in D_{i}} \sum_{j=1}^{M} \int_{D_{j}}\left|\left(g(x, y)-g\left(x_{i}, y\right)\right) p(y) u(y)\right| d y \\
& \leq\|u\|_{\infty}\|p\|_{\infty} \max _{i} \sup _{\left|x-x_{j}\right| \leq \frac{1}{m P}} \sum_{j=1}^{M} \int_{D_{j}}\left|g(x, y)-g\left(x_{i}, y\right)\right| d y \\
& \leq O(1 /(m P)) .
\end{aligned}
$$

This implies

$$
\left\|T-T_{M}\right\|=O(1 /(m P))=O\left(1 / M^{1 / 3}\right) \rightarrow 0 \text { as } M \rightarrow \infty .
$$


The operator $I+T$ is known to be boundedly invertible if $k>0$, so

$$
\left\|(I+T)^{-1}\right\|<c,
$$

where $c>0$ is a constant. Therefore,

$$
I+T_{M}=(I+T)\left[I+(I+T)^{-1}\left(T_{M}-T\right)\right] .
$$

By (54) there exists $M_{0}$ such that

$$
\left\|(I+T)^{-1}\left(T_{M}-T\right)\right\| \leq c\left\|T_{M}-T\right\|<\delta<1, \quad \forall M>M_{0},
$$

where $\delta>0$ is a constant. From (56) we obtain, $\forall M>M_{0}$,

$$
\left\|\left[I+(I+T)^{-1}\left(T_{M}-T\right)\right]^{-1}\right\| \leq \frac{1}{1-\left\|(I+T)^{-1}\left(T_{M}-T\right)\right\|} \leq 1 /(1-\delta) .
$$

Therefore, it follows from (55) that $I+T_{M}$ is boundedly invertible and

$$
\left(I+T_{M}\right)^{-1}=\left[I+(I+T)^{-1}\left(T_{M}-T\right)\right]^{-1}(I+T)^{-1},
$$

so there exists a constant $c_{0}>0$ such that

$$
\left\|\left(I+T_{M}\right)^{-1}\right\| \leq c_{0}, \quad \forall M>M_{0} .
$$

Since (49) is equivalent to (46), it follows that the homogeneous equation

$$
v+T_{d, M} v=0
$$

has only trivial solution for $M>M_{0}$, i.e., $\mathcal{N}\left(I_{d, M}+T_{d, M}\right)=\{0\}$ for $M>M_{0}$, where $\mathcal{N}(A)$ is the nullspace of the operator $A, I_{d, M}$ is the identity operator in $\mathbb{C}^{M}$ and $T_{d, M}$ is defined in (48). Therefore, by the Fredholm alternative equation (46) is solvable for $M>M_{0}$. This together with (59) yield the existence of a constant $c_{1}>0$ such that

$$
\left\|\left(I_{d, M}+T_{d, M}\right)^{-1}\right\| \leq c_{1} \text { for } M>M_{0} .
$$

Lemma 3.1 is proved.

Define $T_{d}: C^{2}(D) \rightarrow \mathbb{C}^{M}$ as follows

$$
\left(T_{d} w\right)_{l}:=(T w)\left(x_{l}\right)=\sum_{j=1}^{M} \int_{D_{j}} g\left(x_{l}, y\right) p(y) w(y) d y, \quad l=1,2, \ldots, M,
$$

and

$$
u_{M}:=\left(\begin{array}{c}
u\left(x_{1}\right) \\
u\left(x_{2}\right) \\
\vdots \\
u\left(x_{M}\right)
\end{array}\right) \in \mathbb{C}^{M}
$$


where $T$ is defined in (40) and $u(x)$ solves (39). Then it follows from (39), (60) and (61) that the following equation holds

$$
u_{M}+T_{d} u=u_{0, M},
$$

where $u_{0, M}$ is defined in (47). Using equations (46) and (62), we derive the following equality:

$$
\begin{aligned}
\left(I_{d, M}+T_{d, M}\right)\left(\tilde{u}_{(M)}-u_{M}\right) & =\left(I_{d, M}+T_{d, M}\right) \tilde{u}_{(M)}-\left(I_{d, M}+T_{d, M}\right) u_{M} \\
& =u_{0, M}-\left(I_{d, M} u_{M}+T_{d, M} u_{M}\right) \\
& =u_{0, M}-u_{M}-T_{d, M} u_{M}=T_{d} u-T_{d, M} u_{M},
\end{aligned}
$$

where $\tilde{u}_{(M)}$ and $u_{0, M}$ are defined in (47),

$$
I_{d, M} v=v, \quad \forall v=\left(\begin{array}{c}
v_{1} \\
v_{2} \\
\vdots \\
v_{M}
\end{array}\right) \in \mathbb{C}^{M}
$$

Using relation (63), one gets

$$
\tilde{u}_{(M)}-u_{M}=\left(I_{d, M}+T_{d, M}\right)^{-1}\left(T_{d} u-T_{d, M} u_{M}\right) .
$$

Lemma 3.2: Let Assumption A) hold (see Section 2 below (25)). Suppose u(x) solves (39) and $p(x) \in C^{1}$. Then

$$
\left\|\tilde{u}_{(M)}-u_{M}\right\|_{\mathbb{C}^{M}}=O\left(1 / M^{2 / 3}\right) \text { as } M \rightarrow \infty,
$$

where $u_{M}$ and $\tilde{u}_{(M)}$ are defined in (61) and (47), respectively.

Proof: By (65) and estimate (52) we obtain

$$
\begin{aligned}
\left\|\tilde{u}_{(M)}-u_{M}\right\|_{\mathbb{C}^{M}} & \leq\left\|\left(I_{d, M}+T_{d, M}\right)^{-1}\right\|\left\|T_{d} u-T_{d, M} u_{M}\right\|_{\mathbb{C}^{M}} \\
& \leq c_{1}\left\|T_{d} u-T_{d, M} u_{M}\right\|_{\mathbb{C}^{M}},
\end{aligned}
$$

where $T_{d, M}$ and $T_{d}$ are defined in (48) and (60), respectively. Let us derive an estimate for $\left\|T_{d} u-T_{d, M} u_{M}\right\|_{\mathbb{C}^{M}}$. We have

$$
\begin{aligned}
\left\|T_{d} u-T_{d, M} u_{M}\right\|_{\mathbb{C}^{M}} & =\max _{1 \leq l \leq M}\left|\sum_{j=1}^{M} \int_{D_{j}} g\left(x_{l}, y\right) p(y)\left(u(y)-u\left(x_{j}\right)\right) d y\right| \\
& \leq J_{1}+J_{2},
\end{aligned}
$$

where

$$
J_{1}:=\max _{1 \leq l \leq M}\left|\int_{D_{l}} g\left(x_{l}, y\right) p(y)\left(u(y)-u\left(x_{l}\right)\right) d y\right|
$$


and

$$
J_{2}:=\max _{1 \leq l \leq M}\left|\sum_{j=1, j \neq l}^{M} \int_{D_{j}} g\left(x_{l}, y\right) p(y)\left(u(y)-u\left(x_{j}\right)\right) d y\right| .
$$

Since $u(x), p(x) \in C(D)$, we get

$$
\begin{aligned}
J_{1} & \leq 2\|p\|_{\infty}\|u\|_{\infty} \max _{1 \leq l \leq M} \int_{D_{l}} \frac{1}{4 \pi\left|x_{l}-y\right|} d y \leq c(p, u) \int_{0}^{\sqrt{3} /(2 m P)} r d r \\
& =\frac{3 c(p, u)}{2(2 m P)^{2}}
\end{aligned}
$$

where $c(p, u):=2\|p\|_{\infty}\|u\|_{\infty}$. Using the identity

$$
u(y)-u\left(x_{j}\right)=u(y)-u\left(x_{j}\right)-\mathcal{D} u\left(x_{j}\right)\left(y-x_{j}\right)+\mathcal{D} u\left(x_{j}\right)\left(y-x_{j}\right)
$$

and applying the triangle inequality, we get the estimate

$$
J_{2} \leq I_{1}+I_{2}
$$

where

$$
I_{1}:=\max _{1 \leq l \leq M}\left|\sum_{j=1, j \neq l}^{M} \int_{D_{j}} g\left(x_{l}, y\right) p(y)\left(u(y)-u\left(x_{j}\right)-\mathcal{D} u\left(x_{j}\right)\left(y-x_{j}\right)\right) d y\right|
$$

and

$$
I_{2}:=\max _{1 \leq l \leq M}\left|\sum_{j=1, j \neq l}^{M} \int_{D_{j}} g\left(x_{l}, y\right) p(y) \mathcal{D} u\left(x_{j}\right)\left(y-x_{j}\right) d y\right| .
$$

Let us derive an estimate for $I_{1}$. Using the Taylor expansion, one gets

$$
I_{1} \leq\|p\|_{\infty} \max _{1 \leq l \leq M} \sum_{j=1, j \neq l}^{M} \int_{D_{j}}\left|g\left(x_{l}, y\right)\right| \sup _{0 \leq s \leq 1}\left|\mathcal{D}^{2} u\left(s y+(1-s) x_{j}\right) \| y-x_{j}\right|^{2} d y
$$

Since $p \in C^{1}(D), u \in C^{2}(D), \int_{D}|g(x, y)| d y<\infty$ and $\left|y-x_{j}\right| \leq \frac{\sqrt{3}}{2 m P}$ for $y \in D_{j}$, it follows from (73) that

$$
I_{1}=O\left(1 /(m P)^{2}\right)=O\left(1 / M^{2 / 3}\right), \quad \text { as } M \rightarrow \infty
$$

Estimate of $I_{2}$ is obtained as follows. Since $x_{j}$ is the centre of the cube $D_{j}$, one has

$$
\int_{D_{j}}\left(y-x_{j}\right) d y=0
$$


so it follows that

$$
\int_{D_{j}} g\left(x_{l}, x_{j}\right) p\left(x_{j}\right) \mathcal{D} u\left(x_{j}\right)\left(y-x_{j}\right) d y=0, \quad j=1,2, \ldots, M .
$$

Therefore, using (75), $I_{2}$ can be rewritten as follows:

$$
I_{2}=\max _{1 \leq l \leq M}\left|\sum_{j=1, j \neq l}^{M} \int_{D_{j}}\left(g\left(x_{l}, y\right) p(y)-g\left(x_{l}, x_{j}\right) p\left(x_{j}\right)\right) \mathcal{D} u\left(x_{j}\right)\left(y-x_{j}\right) d y\right| .
$$

Let

$$
g_{l}(y):=g\left(x_{l}, y\right), \quad\left(g_{l} p\right)(y)=g_{l}(y) p(y), \quad l=1,2, \ldots, M .
$$

Then the formulas

$$
\begin{aligned}
\left|\left(g_{l} p\right)(y)-\left(g_{l} p\right)\left(x_{j}\right)\right| & =\left|\int_{0}^{1} \frac{\partial}{\partial t}\left(g_{l} p\right)\left(t y+(1-t) x_{j}\right) d t\right| \\
& \leq \sup _{0 \leq t \leq 1}\left|\mathcal{D}_{y}\left(g_{l} p\right)\left(t y+(1-t) x_{j}\right)\right|\left|y-x_{j}\right|
\end{aligned}
$$

and

$$
\mathcal{D}_{y}\left(g_{l} p\right)(y)=p(y) \mathcal{D}_{y} g_{l}(y)+g_{l}(y) \mathcal{D} p(y),
$$

yield the following estimate:

$$
\begin{aligned}
I_{2} \leq & \frac{\sqrt{3}\|\mathcal{D} u\|_{\infty}}{2 m P} \max _{1 \leq l \leq M}\|p\|_{\infty} \int_{D} \sup _{0 \leq t \leq 1}\left|\mathcal{D}_{y} g_{l}\left(t y+(1-t) x_{j}\right)\right|\left|y-x_{j}\right| d y \\
& +\frac{\sqrt{3}\|\mathcal{D} u\|_{\infty}}{2 m P} \max _{1 \leq l \leq M}\|\mathcal{D} p\|_{\infty} \int_{D} \sup _{0 \leq t \leq 1}\left|g_{l}\left(t y+(1-t) x_{j}\right) \| y-x_{j}\right| d y \\
\leq & \frac{c(k)\|\mathcal{D} u\|_{\infty}}{(m P)^{2}} \max _{1 \leq l \leq M}\|p\|_{\infty} \int_{D}\left(\frac{1}{4 \pi\left|x_{l}-y\right|}+\frac{1}{4 \pi\left|x_{l}-y\right|^{2}}\right) d y \\
& +\frac{\tilde{c}\|\mathcal{D} u\|_{\infty}}{(m P)^{2}} \max _{1 \leq l \leq M}\|\mathcal{D} p\|_{\infty} \int_{D} \frac{1}{4 \pi\left|x_{l}-y\right|} d y=O\left(1 /(m P)^{2}\right) \\
= & O\left(1 / M^{2 / 3}\right),
\end{aligned}
$$

where $\tilde{c}>0$ is a constant and $c(k)$ is a constant depending on the wave number $k$. Here the estimates $\left|y-x_{j}\right| \leq \sqrt{3} /(2 m P)$ for $y \in D_{j}, \int_{D} \frac{1}{4 \pi\left|x_{l}-y\right|^{\beta}} d y<\infty$ for $\beta<3$, and $\left|x_{l}-y\right| \leq 2\left|x_{l}-s\right|$ for $y \in D_{j}, j \neq l, s=t x_{j}+(1-t) y, t \in[0,1]$, were used. The relation (66) follows from (67), (68), (69), (70), (74) and (79).

Lemma 3.2 is proved.

Lemma 3.3: Let the Assumption A) hold. Consider the linear algebraic system for the unknowns $u_{e}\left(x_{l}\right)$ :

$$
u_{e}\left(x_{l}\right)=u_{0}\left(x_{l}\right)-4 \pi \sum_{j=1, j \neq l}^{M} g\left(x_{l}, x_{j}\right) h\left(x_{j}\right) a_{m P}^{2-\kappa} u_{e}\left(x_{j}\right), \quad l=1,2, \ldots, M,
$$


where $p(x)=4 \pi h(x) N(x) \in C^{2}(D), \quad N(x)=1 / \gamma^{3}, \quad M=(m P)^{3}$, and $g(x, y)$ is defined in (40). Then

$$
\left\|\tilde{u}_{(M)}-u_{e, M}\right\|_{\mathbb{C}^{M}}=O\left(\frac{\log M}{M^{2 / 3}}+\left|1-\gamma^{3} M a_{m P}^{2-\kappa}\right|\right) \quad \text { as } M \rightarrow \infty,
$$

where $\tilde{u}_{(M)}$ is defined in (47),

$$
u_{e, M}:=\left(\begin{array}{c}
u_{e}\left(x_{1}\right) \\
u_{e}\left(x_{2}\right) \\
\vdots \\
u_{e}\left(x_{M}\right)
\end{array}\right) \in \mathbb{C}^{M}
$$

and $u_{e}\left(x_{j}\right), j=1,2, \ldots, M$, solve system $(80)$.

Proof: Let us rewrite (80) as

$$
\begin{aligned}
u_{e}\left(x_{l}\right) & =u_{0}\left(x_{l}\right)-\sum_{j=1, j \neq l}^{M} g\left(x_{l}, x_{j}\right) p\left(x_{j}\right) \frac{a_{m P}^{2-\kappa}}{N\left(x_{j}\right)\left|D_{j}\right|} u_{e}\left(x_{j}\right)\left|D_{j}\right| \\
& =u_{0}\left(x_{l}\right)-\left(T_{e} u_{e, M}\right)_{l}, \quad l=1,2, \ldots, M
\end{aligned}
$$

where $p(x)=4 \pi h(x) N(x),\left|D_{j}\right|=1 /(m P)^{3}$ is the volume of the cube $D_{j}, N(x)=$ $1 / \gamma^{3}$, and

$$
\left(T_{e} v\right)_{l}:=\sum_{j=1, j \neq l}^{M} g\left(x_{l}, x_{j}\right) p\left(x_{j}\right)\left(\gamma m P a_{m P}^{(2-\kappa) / 3}\right)^{3}\left|D_{j}\right| v_{j}, \quad v=\left(\begin{array}{c}
v_{1} \\
v_{2} \\
\vdots \\
v_{M}
\end{array}\right) \in \mathbb{C}^{M}
$$

$l=1,2, \ldots, M$.

Let us derive an estimate for $\left\|\tilde{u}_{(M)}-u_{e, M}\right\|_{\mathbb{C}^{M}}$. Using equations (46) and (83), we obtain

$$
\begin{aligned}
\left(I_{d, M}+T_{d, M}\right)\left(\tilde{u}_{(M)}-u_{e, M}\right) & =\left(I_{d, M}+T_{d, M}\right) \tilde{u}_{(M)}-\left(I_{d, M}+T_{d, M}\right) u_{e, M} \\
& =u_{0, M}-u_{e, M}-T_{d, M} u_{e, M} \\
& =T_{e} u_{e, M}-T_{d, M} u_{e, M}
\end{aligned}
$$

where $\tilde{u}_{(M)}$ and $u_{0, M}$ are defined in (47), $u_{e, M}, T_{d, M}$ and $T_{e}$ are defined in (82), (48) and (84), respectively. Relation (85) implies

$$
\begin{aligned}
\left\|\tilde{u}_{(M)}-u_{e, M}\right\|_{\mathbb{C}^{M}} & \leq\left\|\left(I_{d, M}+T_{d, M}\right)^{-1}\right\|\left\|T_{e} u_{e, M}-T_{d, M} u_{e, M}\right\|_{\mathbb{C}^{M}} \\
& \leq c_{1}\left\|T_{e} u_{e, M}-T_{d, M} u_{e, M}\right\|_{\mathbb{C}^{M}},
\end{aligned}
$$

where estimate (52) was used. 
Let us derive an estimate for $\left\|T_{e} u_{e, M}-T_{d, M} u_{e, M}\right\|_{\mathbb{C}^{M}}$. Using definitions (48) and (84), one gets

$$
\begin{aligned}
\left\|\left(T_{e}-T_{d, M}\right) u_{e, M}\right\|_{\mathbb{C}^{M}} \\
=\max _{1 \leq l \leq M}\left|\sum_{j=1, j \neq l}^{M} g_{l}\left(x_{j}\right) p_{a_{m P}}\left(x_{j}\right) u_{e}\left(x_{j}\right)\right| D_{j}\left|-\sum_{j=1}^{M} \int_{D_{j}} g_{l}(y) p(y) d y u_{e}\left(x_{j}\right)\right| \\
=\max _{1 \leq l \leq M} \mid \sum_{j=1, j \neq l}^{M} \int_{D_{j}}\left(g_{l}\left(x_{j}\right) p_{a_{m P}}\left(x_{j}\right)-g_{l}(y) p(y)\right) d y u_{e}\left(x_{j}\right) \\
\quad-\int_{D_{l}} g_{l}(y) p(y) d y u_{e}\left(x_{l}\right) \mid
\end{aligned}
$$

Applying the triangle inequality to the above equation, we obtain

$$
\begin{aligned}
\left\|\left(T_{e}-T_{d, M}\right) u_{e, M}\right\|_{\mathbb{C}^{M}} \leq & \max _{1 \leq l \leq M}\left|\int_{D_{l}} g_{l}(y) p(y) u_{e}\left(x_{l}\right) d y\right| \\
& +\max _{1 \leq l \leq M}\left|\sum_{j=1, j \neq l}^{M} \int_{D_{j}} g_{l}(y)\left(p(y)-p\left(x_{j}\right)\right) u_{e}\left(x_{j}\right) d y\right| \\
& +\max _{1 \leq l \leq M}\left|\sum_{j=1, j \neq l}^{M} \int_{D_{j}} g_{l}(y)\left(p\left(x_{j}\right)-p_{a_{m P}}\left(x_{j}\right)\right) u_{e}\left(x_{j}\right) d y\right| \\
& +\max _{1 \leq l \leq M}\left|\sum_{j=1, j \neq l}^{M} p_{a_{m P}}\left(x_{j}\right) \int_{D_{j}}\left(g_{l}(y)-g_{l}\left(x_{j}\right)\right) u_{e}\left(x_{j}\right) d y\right| \\
\leq & \max _{1 \leq l \leq M}\left(J_{0}(l)+J_{1}(l)+J_{2}(l)+J_{3}(l)\right)
\end{aligned}
$$

where $g_{l}(y):=g\left(x_{l}, y\right), p_{a_{m P}}(x):=p(x)\left(\gamma m P a_{m P}^{(2-\kappa) / 3}\right)^{3}$,

$$
\begin{aligned}
& J_{0}(l):=\int_{D_{l}}\left|g\left(x_{l}, y\right) p(y) u_{e}\left(x_{l}\right)\right| d y, \\
& J_{1}(l):=\sum_{j=1, j \neq l}^{M}\left|\int_{D_{j}} g\left(x_{l}, y\right)\left(p(y)-p\left(x_{j}\right)\right) u_{e}\left(x_{j}\right) d y\right|, \\
& J_{2}(l):=\sum_{j=1, j \neq l}^{M} \int_{D_{j}}\left|g\left(x_{l}, y\right)\left(p\left(x_{j}\right)-p_{a_{m P}}\left(x_{j}\right)\right) u_{e}\left(x_{j}\right)\right| d y,
\end{aligned}
$$

and

$$
J_{3}(l):=\sum_{j=1, j \neq l}^{M}\left|p_{a_{m P}}\left(x_{j}\right)\right|\left|\int_{D_{j}}\left(g\left(x_{l}, y\right)-g\left(x_{l}, x_{j}\right)\right) u_{e}\left(x_{j}\right) d y\right|
$$


Using the estimate $\left|x_{l}-y\right| \leq \sqrt{3} /(2 m P)$ for $y \in D_{l}$, one gets the following estimate of $J_{0}(l)$ :

$$
\begin{aligned}
J_{0}(l) & \leq\|p\|_{\infty}\left\|u_{e}\right\|_{\mathbb{C}^{M}} \int_{D_{l}}\left|g\left(x_{l}, y\right)\right| d y \\
& \leq\left(\int_{B_{\sqrt{3} /(2 m P)}\left(x_{l}\right)} \frac{1}{4 \pi\left|x_{l}-y\right|} d y\right)\|p\|_{\infty}\left\|u_{e}\right\|_{\mathbb{C}^{M}} \\
& =\left(\int_{0}^{\sqrt{3} /(2 m P)} r d r\right)\|p\|_{\infty}\left\|u_{e}\right\|_{\mathbb{C}^{M}} \\
& =\frac{3\|p\|_{\infty}\left\|u_{e}\right\|_{\mathbb{C}^{M}}}{2(2 m P)^{2}}=O\left(1 / M^{2 / 3}\right),
\end{aligned}
$$

where $B_{a}(x)$ is defined in (19).

Let us estimate $J_{1}(l)$. Using the identity

$$
p(y)-p\left(x_{j}\right)=p(y)-p\left(x_{j}\right)-\mathcal{D} p\left(x_{j}\right) \cdot\left(y-x_{j}\right)+\mathcal{D} p\left(x_{j}\right) \cdot\left(y-x_{j}\right)
$$

in (89) and applying the triangle inequality, one obtains

$$
J_{1}(l) \leq J_{1,1}+J_{1,2},
$$

where

$$
J_{1,1}:=\sum_{j=1, j \neq l}^{M}\left|\int_{D_{j}} g\left(x_{l}, y\right)\left[p(y)-p\left(x_{j}\right)-\mathcal{D} p\left(x_{j}\right) \cdot\left(y-x_{j}\right)\right] u_{e}\left(x_{j}\right) d y\right|,
$$

and

$$
J_{1,2}:=\sum_{j=1, j \neq l}^{M}\left|\int_{D_{j}} g\left(x_{l}, y\right) \mathcal{D} p\left(x_{j}\right) \cdot\left(y-x_{j}\right) u_{e}\left(x_{j}\right) d y\right| .
$$

To get an estimate for $J_{1,1}$, we apply the Taylor expansion of $p(x)$ and get

$$
\begin{aligned}
J_{1,1} & \leq \frac{\left\|u_{e}\right\|_{\mathbb{C}^{M}}}{2} \sum_{j=1, j \neq l}^{M} \int_{D_{j}}\left|g\left(x_{l}, y\right)\right| \sup _{0 \leq t \leq 1}\left|\mathcal{D}^{2} p\left(t y+(1-t) x_{j}\right) \| y-x_{j}\right|^{2} d y \\
& \leq \frac{3\left\|\mathcal{D}^{2} p\right\|_{\infty}\left\|u_{e}\right\|_{\mathbb{C}^{M}}}{8(m P)^{2}} \sum_{j=1, j \neq l}^{M} \int_{D_{j}}\left|g\left(x_{l}, y\right)\right| d y \\
& =O\left(1 /(m P)^{2}\right)=O\left(1 / M^{2 / 3}\right) \text { as } M \rightarrow \infty,
\end{aligned}
$$

where $B_{a}(x)$ is defined in (19), and the estimate $\left|y-x_{j}\right| \leq \sqrt{3} /(2 m P), y \in D_{j}$, was used.

Using the identity

$$
\int_{D_{j}} g\left(x_{l}, x_{j}\right) \mathcal{D} p\left(x_{j}\right)\left(y-x_{j}\right) u_{e}\left(x_{j}\right) d y=0, \quad j=1,2, \ldots, M,
$$


one gets

$$
J_{1,2}=\sum_{j=1, j \neq l}^{M}\left|\int_{D_{j}}\left(g\left(x_{l}, y\right)-g\left(x_{l}, x_{j}\right)\right) \mathcal{D} p\left(x_{j}\right) \cdot\left(y-x_{j}\right) u_{e}\left(x_{j}\right) d y\right| .
$$

Using the assumption $p \in C^{1}(D)$, one derives the following estimate of $J_{1,2}$ :

$$
\begin{aligned}
J_{1,2} \leq & \left\|u_{e}\right\|_{\mathbb{C}^{M}} \sum_{j=1, j \neq l}^{M} \int_{D_{j}}\left|\left(g\left(x_{l}, y\right)-g\left(x_{l}, x_{j}\right)\right) \mathcal{D} p\left(x_{j}\right) \cdot\left(y-x_{j}\right)\right| d y \\
\leq & \frac{\sqrt{3}\|\mathcal{D} p\|_{\infty}\left\|u_{e}\right\|_{\mathbb{C}^{M}}}{2 m P} \sum_{j=1, j \neq l}^{M} \int_{D_{j}}\left|g\left(x_{l}, y\right)-g\left(x_{l}, x_{j}\right)\right| d y \\
\leq & \frac{\sqrt{3}\|\mathcal{D} p\|_{\infty}\left\|u_{e}\right\|_{\mathbb{C}^{M}}}{2 m P} \sum_{j=1, j \neq l}^{M} \int_{D_{j}} \sup _{0 \leq t \leq 1}\left|\mathcal{D} g\left(x_{l}, t y+(1-t) x_{j}\right) \| y-x_{j}\right| d y \\
\leq & \frac{c(k)\|\mathcal{D} p\|_{\infty}\left\|u_{e}\right\|_{\mathbb{C}^{M}}}{(m P)^{2}} \sum_{j=1, j \neq l}^{M} \int_{D_{j}} \sup _{0 \leq t \leq 1} \frac{1}{4 \pi\left|x_{l}-t y-(1-t) x_{j}\right|^{2}} d y \\
& +\frac{c(k)\|\mathcal{D} p\|_{\infty}\left\|u_{e}\right\|_{\mathbb{C}^{M}}}{(m P)^{2}} \sum_{j=1, j \neq l}^{M} \int_{D_{j}} \sup _{0 \leq t \leq 1} \frac{1}{4 \pi\left|x_{l}-t y-(1-t) x_{j}\right|} d y \\
\leq & \frac{c(k)\|\mathcal{D} p\|_{\infty}\left\|u_{e}\right\|_{\mathbb{C}^{M}}}{(m P)^{2}} \int_{B_{\sqrt{3}}\left(x_{l}\right)}\left(\frac{1}{4 \pi\left|x_{l}-y\right|}+\frac{1}{4 \pi\left|x_{l}-y\right|^{2}}\right) d y \\
= & O\left(1 /(m P)^{2}\right)=O\left(1 / M^{2 / 3}\right) \text { as } M \rightarrow \infty
\end{aligned}
$$

where $c(k)$ is a constant depending on the wave number $k$ and $B_{a}(x)$ is defined in (19). Here the estimates $\left|y-x_{j}\right| \leq \sqrt{3} /(2 m P)$ for $y \in D_{j}, \int_{D} \frac{1}{4 \pi\left|x_{l}-y\right|^{\beta}} d y<\infty$ for $\beta<3$, and $\left|x_{l}-y\right| \leq 2\left|x_{l}-s\right|$ for $y \in D_{j}, j \neq l, s=t x_{j}+(1-t) y, t \in[0,1]$, were used. Applying estimates (97) and (99) to (94), we get

$$
J_{1}(l)=O\left(1 / M^{2 / 3}\right), \quad \text { as } M \rightarrow \infty .
$$

Let us derive an estimate for $J_{2}(l)$. From $(90)$ and the definition $p_{a_{m P}}(x)=$ $p(x)\left(\gamma m P a^{(2-\kappa) / 3}\right)^{3}$ we get

$$
\begin{aligned}
J_{2}(l) & \leq\left\|u_{e}\right\|_{\mathbb{C}^{M}} \sum_{j=1, j \neq l}^{M} \int_{D_{j}}\left|g\left(x_{l}, y\right)\left\|p\left(x_{j}\right)\right\| 1-\left(\gamma m P a^{(2-\kappa) / 3}\right)^{3}\right| d y \\
& \leq\left\|u_{e}\right\|_{\mathbb{C}^{M}}\|p\|_{\infty}\left|1-\left(\gamma m P a^{(2-\kappa) / 3}\right)^{3}\right| \sum_{j=1, j \neq l}^{M} \int_{D_{j}}\left|g\left(x_{l}, y\right)\right| d y \\
& \leq\left\|u_{e}\right\|_{\mathbb{C}^{M}}\|p\|_{\infty}\left|1-\left(\gamma m P a^{(2-\kappa) / 3}\right)^{3}\right| \int_{B_{\sqrt{3}}\left(x_{l}\right)} \frac{1}{4 \pi\left|x_{l}-y\right|} d y \\
& =\left(\int_{0}^{\sqrt{3}} r d r\right)\left\|u_{e}\right\|_{\mathbb{C}^{M}}\|p\|_{\infty}\left|1-\left(\gamma m P a^{(2-\kappa) / 3}\right)^{3}\right| \\
& =\frac{3}{2}\left\|u_{e}\right\|_{\mathbb{C}^{M}}\|p\|_{\infty}\left|1-\gamma^{3} M a_{m P}^{(2-\kappa)}\right|=O\left(\left|1-\gamma^{3} M a_{m P}^{(2-\kappa)}\right|\right),
\end{aligned}
$$


where $B_{a}(x)$ is defined in (19). Estimate of $J_{3}(l)$ is derived as follows. Using the identity

$$
\int_{D_{j}} \mathcal{D} g\left(x_{l}, x_{j}\right)\left(y-x_{j}\right) u_{e}\left(x_{j}\right) d y=0, \quad j=1,2, \ldots M
$$

one gets the following estimate:

$$
\begin{aligned}
J_{3}(l)= & \sum_{j=1, j \neq l}^{M}\left|p_{a_{m} P}\left(x_{j}\right)\right| u_{e}\left(x_{j}\right) \int_{D_{j}}\left[g\left(x_{l}, y\right)-g\left(x_{l}, x_{j}\right)-\mathcal{D} g\left(x_{l}, x_{j}\right) \cdot\left(y-x_{j}\right)\right] d y \mid \\
\leq & \frac{\|p\|_{\infty}\left\|u_{e}\right\|_{\mathbb{C}^{M} \gamma^{3} M a_{m P}^{2-\kappa}}}{2} \sum_{j=1, j \neq l}^{M} \int_{D_{j}} \sup _{0 \leq t \leq 1}\left|\mathcal{D}^{2} g\left(x_{l}, t y+(1-t) x_{j}\right)\right|\left|y-x_{j}\right|^{2} d y \\
\leq & \frac{3 c_{M}}{8(m P)^{2}} \sum_{j=1, j \neq l}^{M} \int_{D_{j}} \sup _{0 \leq t \leq 1}\left|\mathcal{D}^{2} g\left(x_{l}, t y+(1-t) x_{j}\right)\right| d y \\
\leq & \frac{c(k) c_{M}}{(m P)^{2}} \sum_{j=1, j \neq l}^{M} \int_{D_{j}} \sup _{0 \leq t \leq 1} \frac{1}{4 \pi\left|x_{l}-t y-(1-t) x_{j}\right|} d y \\
& +\frac{c(k) c_{M}}{(m P)^{2}} \sum_{j=1, j \neq l}^{M} \int_{D_{j}} \sup _{0 \leq t \leq 1} \frac{1}{4 \pi\left|x_{l}-t y-(1-t) x_{j}\right|^{2}} d y \\
& +\frac{c(k) c_{M}}{(m P)^{2}} \sum_{j=1, j \neq l}^{M} \int_{D_{j}} \sup _{0 \leq t \leq 1} \frac{1}{4 \pi\left|x_{l}-t y-(1-t) x_{j}\right|^{3}} d y \\
\leq & \frac{c(k) c_{M}}{(m P)^{2}} \int_{1 /(2 m P)<\left|x_{l}-y\right|<\sqrt{3}}\left(\frac{1}{4 \pi\left|x_{l}-y\right|}+\frac{1}{4 \pi\left|x_{l}-y\right|^{2}}+\frac{1}{4 \pi\left|x_{l}-y\right|^{3}}\right) d y \\
\leq & \frac{c(k) c_{M}}{(m P)^{2}} \int_{1 /(2 m P)}^{\sqrt{3}}\left(r+1+\frac{1}{r}\right) d r \\
\leq & \frac{2 c(k) c_{M}}{(m P)^{2}}[1+\log (\sqrt{3})-\log (1 /(2 m P))] \\
= & \frac{2 c(k) c_{M}}{M^{2 / 3}}\left[1+\log (\sqrt{3})-\log \left(1 /\left(2 M^{1 / 3}\right)\right)\right]=O\left(\frac{\log M}{M^{2 / 3}}\right),
\end{aligned}
$$

where $c_{M}:=\|p\|_{\infty}\left\|u_{e}\right\|_{\mathbb{C}^{M}} \gamma^{3} M a_{m P}^{2-\kappa}, c(k)$ is a constant depending on the wave number $k$. Here the estimates $\left|y-x_{j}\right| \leq \sqrt{3} /(2 m P)$ for $y \in D_{j}$, and $\mid x_{l}-$ $y|\leq 2| x_{l}-s \mid$ for $y \in D_{j}, \quad j \neq l, s=t x_{j}+(1-t) y, t \in[0,1]$, were used. Using estimates (97), (99), (101) and (103), one gets relation (81).

Lemma 3.3 is proved.

The following theorem is a consequence of Lemmas 3.2 and 3.3.

Theorem 3.4: Suppose that the assumptions of Lemmas 3.2 and 3.3 hold. Then

$$
\left\|u_{M}-u_{e, M}\right\|_{\mathbb{C}^{M}}=O\left(\frac{\log M}{M^{2 / 3}}+\left|1-\gamma^{3} M a_{m P}^{2-\kappa}\right|\right) \quad \text { as } M \rightarrow \infty,
$$

where $u_{M}$ and $u_{e, M}$ are defined in (61) and (82), respectively. 
To get the rate of convergence (104) we have assumed that $p(x) \in C^{2}(D)$. If $p(x) \in C(D)$ then the rate given in Theorem 3.4 is no longer valid. The rate of $\left\|u_{M}-u_{e, M}\right\|_{\mathbb{C}^{M}}$ when $p(x) \in C(D)$ is given in the following theorem.

Theorem 3.5: Let Assumption A) hold and $p \in C(D)$ satisfies

$$
|p(x)-p(y)| \leq \omega_{p}(|x-y|), \quad \forall x, y \in D,
$$

where $\omega_{p}$ is the modulus of continuity of the function $p(x)$.Then

$$
\left\|u_{M}-u_{e, M}\right\|_{\mathbb{C}^{M}}=O\left(\frac{\log M}{M^{1 / 3}}+\left|1-\gamma^{3} M a_{m P}^{2-\kappa}\right|+\omega_{p}\left(1 / M^{1 / 3}\right)\right),
$$

where $u_{M}$ and $u_{e, M}$ are defined in (61) and (82), respectively.

Proof: We have

$$
\left\|u_{M}-u_{e, M}\right\|_{\mathbb{C}^{M}} \leq\left\|u_{M}-\tilde{u}_{M}\right\|_{\mathbb{C}^{M}}+\left\|\tilde{u}_{M}-u_{e, M}\right\|_{\mathbb{C}^{M}} .
$$

Let us estimate $\left\|u_{M}-\tilde{u}_{M}\right\|_{\mathbb{C}^{M}}$. From (67) we have

$$
\left\|u_{M}-\tilde{u}_{M}\right\|_{\mathbb{C}^{M}} \leq c_{1}\left\|T_{d} u-T_{d, M} u_{M}\right\|_{\mathbb{C}^{M}},
$$

where $c_{1}$ is defined in (52). Using the similar steps given in (68) we get the following estimate for $\left\|T_{d} u-T_{d, M} u_{M}\right\|_{\mathbb{C}^{M}}$ :

$$
\left\|T_{d} u-T_{d, M} u_{M}\right\|_{\mathbb{C}^{M}} \leq \frac{3\|p\|_{\infty}\|u\|_{\infty}}{2(m P)^{2}}+I_{1}+I_{2},
$$

where $I_{1}$ and $I_{2}$ are defined in (71) and (72), respectively. It is shown in (74) that $I_{1}=O\left(1 / M^{2 / 3}\right)$. Since $p(x) \in C(D)$, the steps (76)-(79) are no longer valid. The estimate of $I_{2}$ can be derived as follows. Since $p \in C(D)$ and $u \in C^{1}(D)$, it follows from (72) that

$$
\begin{aligned}
I_{2} & \leq\|p\|_{\infty}\|\mathcal{D} u\|_{\infty} \max _{1 \leq l \leq M} \sum_{j=1, \neq l}^{M} \int_{D_{j}}\left|g\left(x_{l}, y\right)\right|\left|x_{l}-y\right| d y \\
& \leq \frac{\sqrt{3}\|p\|_{\infty}\|\mathcal{D} u\|_{\infty}}{2 m P} \max _{1 \leq l \leq M} \int_{B_{\sqrt{3}}\left(x_{l}\right)} \frac{1}{4 \pi\left|x_{l}-y\right|} d y \\
& =O(1 /(m P))=O\left(1 / M^{1 / 3}\right) .
\end{aligned}
$$

This together with (109) and $I_{1}=O\left(1 / M^{2 / 3}\right)$ yield

$$
\left\|u_{M}-\tilde{u}_{M}\right\|_{\mathbb{C}^{M}}=O\left(1 / M^{1 / 3}\right) .
$$

Let us estimate $\left\|\tilde{u}_{M}-u_{e, M}\right\|_{\mathbb{C}^{M}}$. From (86) we have

$$
\left\|\tilde{u}_{M}-u_{e, M}\right\|_{\mathbb{C}^{M}} \leq c_{1}\left\|T_{e} u_{e, M}-T_{d, M} u_{e, M}\right\|_{\mathbb{C}^{M}},
$$


where $c_{1}$ is defined in (52). By definitions (48) and (84), and use the triangle inequality, we get

$$
\left\|T_{e} u_{e, M}-T_{d, M} u_{e, M}\right\|_{\mathbb{C}^{M}} \leq J_{1}+J_{2},
$$

where

$$
J_{1}:=\max _{1 \leq l \leq M}\left|\int_{D_{l}} g\left(x_{l}, y\right) p(y) d y u_{e}\left(x_{l}\right) d y\right|
$$

and

$$
J_{2}:=\max _{1 \leq l \leq M}\left|\sum_{j=1, j \neq l}^{M} \int_{D_{j}}\left(g\left(x_{l}, x_{j}\right) p_{a_{m P}}\left(x_{j}\right)-g\left(x_{l}, y\right) p(y)\right) u_{e}\left(x_{j}\right) d y\right|,
$$

$p_{a_{m P}}:=p(x)\left(\gamma m P a^{(2-\kappa) / 3}\right)^{3}$. It is proved in (92) that $J_{1}=O\left(1 / M^{2 / 3}\right)$. The estimate of $J_{2}$ is derived as follows. By the triangle inequality we obtain

$$
J_{2} \leq J_{2,1}+J_{2,2}
$$

where

$$
J_{2,1}:=\max _{1 \leq l \leq M}\left|\sum_{j=1, j \neq l}^{M} \int_{D_{j}}\left(g\left(x_{l}, x_{j}\right)-g\left(x_{l}, y\right)\right) p_{a_{m P}}\left(x_{j}\right) u_{e}\left(x_{j}\right) d y\right|
$$

and

$$
J_{2,2}:=\max _{1 \leq l \leq M}\left\|u_{e}\right\|_{\mathbb{C}^{M}} \sum_{j=1, j \neq l}^{M} \int_{D_{j}}\left|g\left(x_{l}, y\right)\right|\left|p_{a_{m P}}\left(x_{j}\right)-p(y)\right| d y .
$$

It is proved in (103) that

$$
J_{2,1}=O\left(\frac{\log M}{M^{2 / 3}}\right) .
$$

To estimate $J_{2,2}$, we apply the triangle inequality and get

$$
\begin{aligned}
J_{2,2} \leq & \left\|u_{e}\right\|_{\mathbb{C}^{M}} \max _{1 \leq l \leq M} \sum_{j=1, j \neq l}^{M} \int_{D_{j}}\left|g\left(x_{l}, y\right)\right|\left|p_{a_{m P}}\left(x_{j}\right)-p\left(x_{j}\right)\right| d y \\
& +\left\|u_{e}\right\|_{\mathbb{C}^{M}} \max _{1 \leq l \leq M} \sum_{j=1, j \neq l}^{M} \int_{D_{j}}\left|g\left(x_{l}, y\right) \| p\left(x_{j}\right)-p(y)\right| d y \\
\leq & \left|\gamma^{3} M a_{m P}^{2-\kappa}-1\right|\|p\|_{\infty} \max _{1 \leq l \leq M}\left\|u_{e}\right\|_{\mathbb{C}^{M}} \sum_{j=1, j \neq l}^{M} \int_{D_{j}}\left|g\left(x_{l}, y\right)\right| d y \\
& +\max _{j} \sup _{y \in D_{j}} \omega_{p}\left(\left|x_{j}-y\right|\right)\left\|u_{e}\right\|_{\mathbb{C}^{M}} \max _{1 \leq l \leq M} \sum_{j=1, j \neq l}^{M} \int_{D_{j}}\left|g\left(x_{l}, y\right)\right| d y \\
= & O\left(\left|\gamma^{3} M a_{m P}^{2-\kappa}-1\right|+\omega_{p}\left(1 / M^{1 / 3}\right)\right),
\end{aligned}
$$


where $\omega_{p}$ is the modulus of continuity of $p(x)$. This together with $J_{1}=O\left(1 / M^{2 / 3}\right)$ and (119) yield

$$
\left\|\tilde{u}_{M}-u_{e, M}\right\|_{\mathbb{C}^{M}}=O\left(\frac{\log M}{M^{2 / 3}}+\left|\gamma^{3} M a_{m P}^{2-\kappa}-1\right|+\omega_{p}\left(1 / M^{1 / 3}\right)\right) .
$$

Relation (106) follows from (107), (111) and (121).

Theorem 3.5 is proved.

It has been mentioned in the introduction that our main goal is to develop an algorithm for obtaining the minimal number of the embedded small balls which generate a material whose refraction coefficient differs from the desired one by not more than a desired small quantity.

Let us derive an approximation of the desired refraction coefficient $n^{2}(x)$ generated by the embedded small balls. We rewrite the sum in (38) as

$$
\sum_{j=1}^{M} g\left(x, x_{j}\right) p_{a_{m P}}\left(x_{j}\right) u\left(x_{j}\right)\left|D_{j}\right|
$$

where $\left|x-x_{j}\right|>a_{m P}, j=1,2, \ldots, M,\left|D_{j}\right|=1 /(m P)^{3}$ is the volume of the cube $D_{j}$, and

$$
p_{a_{m P}}(x):=4 \pi h(x) N(x)\left(\gamma m P a^{(2-\kappa) / 3}\right)^{3}, \quad N(x)=1 / \gamma^{3} .
$$

Since $\left(\gamma m P a^{(2-\kappa) / 3}\right)^{3} \rightarrow 1$ as $m \rightarrow \infty$, it follows that (122) is a Riemannian sum for the integral $\int_{D} g(x, y) p(y) u(y) d y$, where $p(x)=4 \pi h(x) N(x)$. This motivates us to define the following approximation of the refraction coefficient $n^{2}(x)$ :

$$
n_{a_{m P}}^{2}(x):=n_{0}^{2}(x)-k^{-2} p_{a_{m P}}(x),
$$

where $p_{a_{m P}}$ is defined in (123). We are interested in finding the largest radius $a_{m P}$ (or the smallest $M=(m P)^{3}$ ) such that

$$
e(M):=\max _{1 \leq l \leq M}\left|n^{2}\left(x_{l}\right)-n_{a_{m P}}^{2}\left(x_{l}\right)\right| \leq \delta / k^{2}:=\delta(k),
$$

where $k$ is the wave number, $\delta>0$ is a given small quantity and $n_{a_{m P}}^{2}(x)$ is defined in (124).

An estimate of the error $e(M)$, defined in (125), is given in the following theorem.

Theorem 3.6: Suppose Assumption A) holds and $N(x)=1 / \gamma^{3}$. Then

$$
\max _{1 \leq l \leq(m P)^{3}}\left|n^{2}\left(x_{l}\right)-n_{a_{m P}}^{2}\left(x_{l}\right)\right| \leq k^{-2}\|p\|_{\infty}\left|1-\left(\gamma m P a_{m P}^{(2-\kappa) / 3}\right)^{3}\right|,
$$

where $x_{l}$ is the centre of the lth small ball, $p(x)$ is defined in (41), $n^{2}(x)=n_{0}^{2}(x)-$ $k^{-2} p(x)$, and $n_{a_{m P}}^{2}$ is defined in (124). Consequently,

$$
\lim _{m \rightarrow \infty} \max _{1 \leq l \leq(m P)^{3}}\left|n^{2}\left(x_{l}\right)-n_{a_{m P}}^{2}\left(x_{l}\right)\right|=0 .
$$


Proof: Let

$$
I_{l}:=\left|n^{2}\left(x_{l}\right)-n_{a_{m P}}^{2}\left(x_{l}\right)\right|
$$

Then

$$
\begin{aligned}
I_{l} & =k^{-2}\left|p\left(x_{l}\right)-p\left(x_{l}\right)\left[\gamma m P a_{m P}^{(2-\kappa) / 3}\right]^{3}\right| \leq k^{-2}\left|p\left(x_{l}\right)\right|\left|1-\left[\gamma m P a_{m P}^{(2-\kappa) / 3}\right]^{3}\right| \\
& \leq k^{-2}\|p\|_{\infty}\left|1-\left[\gamma m P a_{m P}^{(2-\kappa) / 3}\right]^{3}\right| .
\end{aligned}
$$

This together with relation (22) yield (127).

Theorem 3.6 is proved.

Using Theorem 3.6 one can calculate the smallest $M$ satisfying (125) by the following algorithm:

\section{Algorithm}

Initialisations: Let the wave number $k$, the constant $\delta>0, n_{0}^{2}(x)$ and $n^{2}(x)$ be given. Fix $P>1, m=m_{0}:=1, \kappa \in(0,1), \gamma>[1 /(2 P)]^{(\kappa+1) / 3}$ and $N(x)=1 / \gamma^{3}$. Partition $D$ into $P^{3}$ cubes $\Omega_{q}, D=\cup_{q=1}^{P^{3}} \overline{\Omega_{q}}, \Omega_{j} \cap \Omega_{i}=\emptyset$ for $j \neq i$, where each cube $\Omega_{j}$ has side length $1 / P$.

Step 1: Solve the equation

$$
\gamma a_{m P}^{(2-\kappa) / 3}+a_{m P}-1 /(m P)=0
$$

for $a_{m P}$.

Step 2: $\quad$ Embed $m^{3}$ small balls of radius $a_{m P}$ in each cube $\Omega_{q}$ so that Assumption A) holds.

Step 3: Compute

$p\left(x_{l}\right)=k^{2}\left(n_{0}^{2}\left(x_{l}\right)-n^{2}\left(x_{l}\right)\right)$

and

$p_{a_{m P}}\left(x_{l}\right)=p\left(x_{l}\right)\left[\gamma m P a_{m P}^{(2-\kappa) / 3}\right]^{3}, \quad l=1,2, \ldots,(m P)^{3}$,

where $x_{l}$ is the centre of the $l$ th small ball and $k$ is the wave number.

Step 4: If $\max _{1 \leq l \leq(m P)^{3}}\left|p\left(x_{l}\right)-p_{a_{m P}}\left(x_{l}\right)\right|>\delta$, then set $m=m+1$ and go to Step 1. Otherwise the number $M=(m P)^{3}$ is the smallest number of the balls embedded in $D$ such that inequality (125) holds, and $a_{m P}$ is the radius of each embedded ball. 


\section{Numerical experiments}

In this section we give the results of the numerical experiments. Suppose the refraction coefficient of the original material in $D$ is $n_{0}^{2}(x)=1$ and the desired refraction coefficients are:

Example 1: $n_{1}^{2}(x)=5$,

Example 2: $n_{2}^{2}(x)=5+\exp \left(-\left|x-x_{0}\right|^{2} /\left(2 \sigma^{2}\right)\right) /(\sqrt{2 \pi} \sigma)$, where $x_{0}=(0.5,0.5,0.5)$ and $\sigma=\frac{\sqrt{3}}{2 M^{1 / 3}}$. Here $M$ is the smallest number of the embedded small balls taken from Example 1.

Example 3: $n_{3}^{2}(x)=1+0.5 \sin \left(x_{1}\right)$, where $x_{1}$ is the first component of the vector $x$,

Example 4: $n_{4}^{2}(x)=1+0.5 \sin \left(100 x_{1}\right)$, where $x_{1}$ is the first component of the vector $x$.

Example 5: $n_{5}^{2}(x)=n_{3}^{2}(x)+i \varepsilon$, where $n_{3}^{2}(x)$ is defined in Example 3, $i=\sqrt{-1}$ and $\varepsilon$ is a small positive number.

By the recipe we choose

$$
p(x)=k^{2}\left(n_{0}^{2}(x)-n^{2}(x)\right)=k^{2}\left(1-n^{2}(x)\right), \quad k>0 .
$$

Let us take

$$
P=11, \quad \kappa=0.99, \quad \gamma=10 \sqrt{k}[1 /(2 P)]^{(1+\kappa) / 3}, \quad m_{0}=1,
$$

where $k$ is the wave number and $m_{0}$ is the initial number of small balls described in the algorithm. Here the parameters $P=11$ and $m_{0}=1$ are chosen so that the approximation error in Lemma 3.2 is at most $c(k) 10^{-4}$, where $c(k)$ is a constant depending on the wave number $k$. We apply the algorithm given in Section 3 to get the minimal total number of small balls embedded in the cube $D$ such that inequality (125) holds for various values of $\delta$, where the quantity $\delta$ was defined in the Algorithm (see the Initialisation and Step 4 of the Algorithm).

The smallest number of the balls embedded in $D$ increases as $\delta$ decreases. The radius $a_{m P}$ and the ratio $a_{m P} / d_{m P}$ decrease as $M$ increases, which agrees with the theory. The results are shown in Tables 1-6. In these tables we define

$$
d_{m P}:=\min _{1 \leq i, j \leq M, i \neq j} \operatorname{dist}\left(B_{a_{m P}}\left(x_{i}\right), B_{a_{m P}}\left(x_{j}\right)\right),
$$

where $B_{a}(x)$ is defined in (19), and

$$
\begin{aligned}
E_{j} & :=\max _{1 \leq l \leq M}\left|n_{j}^{2}\left(x_{l}\right)-n_{j, a_{m P}}^{2}\left(x_{l}\right)\right| \\
& =\max _{1 \leq l \leq M}\left|n_{j}^{2}\left(x_{l}\right)-n_{0}^{2}\left(x_{l}\right)\right|\left|1-\gamma^{3} M a_{m P}^{2-\kappa}\right|,
\end{aligned}
$$

where $n_{j, a_{m P}}^{2}(x):=n_{0}^{2}(x)-k^{-2} p_{j, a_{m P}}(x), p_{j, a_{m P}}(x):=p_{j}(x) \gamma^{3} M a_{m P}^{2-\kappa}, M$ is the smallest total number of small balls embedded in the domain $D, a_{m P}$ is the radius 
Table 1 Example 1

\begin{tabular}{cccccc}
\hline & \multicolumn{5}{c}{$k=1$} \\
\cline { 2 - 6 }$\delta$ & $m$ & $M$ & $a_{m P}$ & $a_{m P} / d_{m P}$ & $E_{1}$ \\
\hline $5.000 \times 10^{-2}$ & 1 & $1.331 \times 10^{3}$ & $3.768 \times 10^{-4}$ & $5.467 \times 10^{-2}$ & $4.953 \times 10^{-2}$ \\
$5.000 \times 10^{-3}$ & 4 & $8.518 \times 10^{4}$ & $6.206 \times 10^{-6}$ & $1.372 \times 10^{-2}$ & $3.276 \times 10^{-3}$ \\
\hline & \multicolumn{5}{c}{$k=5$} \\
\cline { 2 - 6 }$\delta$ & $m$ & $M$ & $a_{m P}$ & $a_{m P} / d_{m P}$ & $E_{1}$ \\
\hline $5.000 \times 10^{-2}$ & 2 & $1.065 \times 10^{4}$ & $4.457 \times 10^{-6}$ & $5.489 \times 10^{-3}$ & $1.177 \times 10^{-3}$ \\
$5.000 \times 10^{-3}$ & 5 & $1.664 \times 10^{5}$ & $2.932 \times 10^{-7}$ & $2.196 \times 10^{-3}$ & $1.935 \times 10^{-4}$ \\
\hline
\end{tabular}

Table 2 Example 2

\begin{tabular}{cccccc}
\hline & \multicolumn{5}{c}{$k=1$} \\
\cline { 2 - 6 }$\delta$ & $m$ & $M$ & $a_{m P}$ & $a_{m P} / d_{m P}$ & $E_{2}$ \\
\hline $5.000 \times 10^{-2}$ & 2 & $1.065 \times 10^{4}$ & $4.852 \times 10^{-5}$ & $2.742 \times 10^{-2}$ & $1.812 \times 10^{-2}$ \\
$5.000 \times 10^{-3}$ & 6 & $2.875 \times 10^{5}$ & $1.862 \times 10^{-6}$ & $9.149 \times 10^{-3}$ & $3.904 \times 10^{-3}$ \\
\hline & \multicolumn{5}{c}{$k=5$} \\
\cline { 2 - 6 }$\delta$ & $m$ & $M$ & $a_{m P}$ & $a_{m P} / d_{m P}$ & $E_{2}$ \\
\hline $5.000 \times 10^{-2}$ & 3 & $3.594 \times 10^{4}$ & $1.337 \times 10^{-6}$ & $3.660 \times 10^{-3}$ & $9.765 \times 10^{-4}$ \\
$5.000 \times 10^{-3}$ & 9 & $9.703 \times 10^{5}$ & $5.116 \times 10^{-8}$ & $1.220 \times 10^{-3}$ & $1.891 \times 10^{-4}$ \\
\hline
\end{tabular}

Table 3 Example 3

\begin{tabular}{|c|c|c|c|c|c|}
\hline \multirow[b]{2}{*}{$\delta$} & \multicolumn{5}{|c|}{$k=1$} \\
\hline & $m$ & $M$ & $a_{m P}$ & $a_{m P} / d_{m P}$ & $E_{3}$ \\
\hline $5.000 \times 10^{-2}$ & 1 & $1.331 \times 10^{3}$ & $3.768 \times 10^{-4}$ & $5.467 \times 10^{-2}$ & $5.052 \times 10^{-3}$ \\
\hline \multirow[t]{2}{*}{$1.000 \times 10^{-4}$} & 8 & $6.815 \times 10^{5}$ & $7.923 \times 10^{-7}$ & $6.862 \times 10^{-3}$ & $8.768 \times 10^{-5}$ \\
\hline & \multicolumn{5}{|c|}{$k=5$} \\
\hline$\delta$ & $m$ & $M$ & $a_{m P}$ & $a_{m P} / d_{m P}$ & $E_{3}$ \\
\hline $5.000 \times 10^{-2}$ & 1 & $1.331 \times 10^{3}$ & $3.490 \times 10^{-5}$ & $1.098 \times 10^{-2}$ & $4.698 \times 10^{-4}$ \\
\hline $1.000 \times 10^{-4}$ & 12 & $2.300 \times 10^{6}$ & $2.177 \times 10^{-8}$ & $9.150 \times 10^{-4}$ & $3.618 \times 10^{-6}$ \\
\hline
\end{tabular}

of the embedded small balls and $x_{l}$ is the centre of the $l$ th small ball. In Example 1 we choose a constant refraction coefficient $n^{2}(x)$. For $k=1$ the total number of small balls $M$ increases by $8.385 \times 10^{4}$ when the error level $\delta$ is decreased by $4.5 \%$, while for $k=5$ the value of $M$ increases by $1.558 \times 10^{5}$ as the error level $\delta$ decreases by $4.5 \%$, as shown in Table 1 .

In Example 2 we add a Gaussian function to the constant refraction coefficient $n_{1}^{2}(x)$ considered in Example 1. Since $\left|n_{0}^{2}(x)-n_{1}^{2}(x)\right| \leq\left|n_{0}^{2}(x)-n_{2}^{2}(x)\right|$, it follows from (133) that $E_{1} \leq E_{2}$. Therefore, in this example one may need to embed more small balls to reach the same error level $\delta$ as in Example 1. The numerical results 
Table 4 Example 4

\begin{tabular}{cccccc}
\hline & \multicolumn{5}{c}{$k=1$} \\
\cline { 2 - 6 }$\delta$ & $m$ & $M$ & $a_{m P}$ & $a_{m P} / d_{m P}$ & $E_{4}$ \\
\hline $5.000 \times 10^{-2}$ & 1 & $1.331 \times 10^{3}$ & $3.768 \times 10^{-4}$ & $5.467 \times 10^{-2}$ & $6.188 \times 10^{-3}$ \\
$1.000 \times 10^{-4}$ & 9 & $9.703 \times 10^{5}$ & $5.584 \times 10^{-7}$ & $6.099 \times 10^{-3}$ & $8.289 \times 10^{-5}$ \\
\hline & \multicolumn{5}{c}{$k=5$} \\
& \multicolumn{5}{c}{$a_{m P} / d_{m P}$} \\
\cline { 2 - 6 }$\delta$ & $m$ & $M$ & $a_{m P}$ & $E_{4}$ \\
\hline $5.000 \times 10^{-2}$ & 1 & $1.331 \times 10^{3}$ & $3.490 \times 10^{-5}$ & $1.098 \times 10^{-2}$ & $5.754 \times 10^{-4}$ \\
$1.000 \times 10^{-4}$ & 13 & $2.924 \times 10^{6}$ & $1.716 \times 10^{-8}$ & $8.446 \times 10^{-4}$ & $3.681 \times 10^{-6}$ \\
\hline
\end{tabular}

Table 5 Example 5 with $\operatorname{Im} n_{5}^{2}(x)=0.02$

\begin{tabular}{|c|c|c|c|c|c|}
\hline \multirow[b]{2}{*}{$\delta$} & \multicolumn{5}{|c|}{$k=1$} \\
\hline & $m$ & $M$ & $a_{m P}$ & $a_{m P} / d_{m P}$ & $E_{5}$ \\
\hline $5.000 \times 10^{-2}$ & 1 & $1.331 \times 10^{3}$ & $3.490 \times 10^{-5}$ & $1.098 \times 10^{-2}$ & $4.703 \times 10^{-4}$ \\
\hline \multirow[t]{2}{*}{$1.000 \times 10^{-4}$} & 8 & $6.815 \times 10^{5}$ & $7.923 \times 10^{-7}$ & $6.862 \times 10^{-3}$ & $8.778 \times 10^{-5}$ \\
\hline & \multicolumn{5}{|c|}{$k=5$} \\
\hline$\delta$ & $m$ & $M$ & $a_{m P}$ & $a_{m P} / d_{m P}$ & $E_{5}$ \\
\hline $5.000 \times 10^{-2}$ & 1 & $1.331 \times 10^{3}$ & $3.490 \times 10^{-5}$ & $1.098 \times 10^{-2}$ & $4.703 \times 10^{-4}$ \\
\hline $1.000 \times 10^{-4}$ & 12 & $2.300 \times 10^{6}$ & $2.177 \times 10^{-8}$ & $9.150 \times 10^{-4}$ & $3.622 \times 10^{-6}$ \\
\hline
\end{tabular}

Table 6 Example 5 with $\operatorname{Im} n_{5}^{2}(x)=0.2$

\begin{tabular}{cccccc}
\hline & \multicolumn{5}{c}{$k=1$} \\
\cline { 2 - 6 }$\delta$ & $m$ & $M$ & $a_{m P}$ & $a_{m P} / d_{m P}$ & $E_{5}$ \\
\hline $5.000 \times 10^{-2}$ & 1 & $1.331 \times 10^{3}$ & $3.768 \times 10^{-4}$ & $5.467 \times 10^{-2}$ & $5.626 \times 10^{-3}$ \\
$1.000 \times 10^{-4}$ & 8 & $6.815 \times 10^{5}$ & $7.923 \times 10^{-7}$ & $6.862 \times 10^{-3}$ & $9.714 \times 10^{-5}$ \\
\hline & \multicolumn{5}{c}{$k=5$} \\
& \multicolumn{5}{c}{$a_{m P} / d_{m P}$} \\
\cline { 2 - 6 }$\delta$ & $m$ & $M$ & $a_{m P}$ & $E_{5}$ \\
\hline $5.000 \times 10^{-2}$ & 1 & $1.331 \times 10^{3}$ & $3.490 \times 10^{-5}$ & $1.098 \times 10^{-2}$ & $5.232 \times 10^{-4}$ \\
$1.000 \times 10^{-4}$ & 13 & $2.924 \times 10^{6}$ & $1.716 \times 10^{-8}$ & $8.446 \times 10^{-4}$ & $3.424 \times 10^{-6}$ \\
\hline
\end{tabular}

show that the values of $M$ in Example 2 are higher than the values of $M$ of Example 1, see Table 2. The refraction coefficients $n^{2}(x)$ considered in Examples 3 and 4 are periodic. In Example 3 for $k=1$ the total number of the embedded small particles $M$ increases by $6.802 \times 10^{5}$ as the error level $\delta$ decreases by $5 \%$. A significant increment of $M$ is obtained for $k=5$. These results are shown in Table 3.

In Example 4 the angular frequency of the sine function is 100 times the angular frequency of the sine function given in Example 3. In this case we get that for $k=1$ the value of $M$ increases by $9.690 \times 10^{5}$ as the error level $\delta$ decreases by $5 \%$ which 
is higher than the increment given in Example 3. Similarly, for $k=5$ the increment of $M$ in this example is higher than the increment of $M$ obtained in Example 3.

In Example 5 we add a small positive imaginary part to the refraction coefficient $n_{3}^{2}(x)$ defined in Example 3. We observe that if the value of the small imaginary $\varepsilon$ is chosen from the interval $(0,0.2)$ then the values of $M$ obtained in this example are equal to the values of $M$ obtained in Example 3. For simplicity we show only the results of $n_{5}^{2}(x)$ with $\operatorname{Im} n_{5}^{2}(x)=0.02$ in Table 5. The value of $M$ starts increasing when $k=5$ and the value of the small positive imaginary part $\varepsilon$ is equal to 0.2 as shown in Table 6 .

\section{References}

Ramm, A.G. (2007a) 'Many body wave scattering by small bodies and applications', J. Math. Phys., Vol. 48, No. 10, pp.103511.

Ramm, A.G. (2007b) 'Material with the desired refraction coefficients can be made by embedding small particles', Phys. Lett. A, Vol. 370, Nos. 5-6, pp.522-527.

Ramm, A.G. (2008a) 'A recipe for making materials with negative refraction in acoustic', Phys. Lett. A, Vol. 372, No. 13, pp.2319-2321.

Ramm, A.G. (2008b) 'Wave scattering by many small particles embedded in a medium', Phys. Lett. A, Vol. 372, No. 17, pp.3064-3070.

Ramm, A.G. (2009) 'A collocation method for solving integral equations', Internat. Journ. of Comput. Sci. and Math., Vol. 2, No. 3, pp.222-228. 